\title{
The Effect of Long-term Steroid Therapy on Patients Treated with Clofazimine (Lamprene)
}

\author{
L. M. HOGERZEIL AND N. PRABHUDAS \\ Victoria Hospital, Dichpalli 503 175, \\ Nizamabad District, Andhra Pradesh, India
}

\begin{abstract}
A study was undertaken in 18 leprosy patients and 31 controls to find out if Lamprene can prevent a flare-up of the infection with Myco. leprae during long-term steroid therapy. All patients were either BL or LL; some were given daily Lamprene $100 \mathrm{mg}$ and prednisone $10 \mathrm{mg}$ because of persistent reactions, others twice weekly Lamprene $100 \mathrm{mg}$ and daily prednisone $10 \mathrm{mg}$ for the same reason. The duration of treatment varied from 6 to 23 months. The control patients were given Lamprene only, either daily $100 \mathrm{mg}$ or twice weekly $100 \mathrm{mg}$. Their reactions were less severe and therefore they did not require prednisone. It was found that in both groups (Lamprene plus prednisone and Lamprene only) there was a satisfactory reduction of the MI (Morphological Index) to zero or less than $1 \%$. But as regards the BI (Bacteriological Index) the patients on Lamprene plus prednisone did clearly better than the patients on Lamprene only. It thus appears that long-term steroid therapy has no adverse effect on the BI and MI of lepromatous patients, provided that they are treated with Lamprene at the same time.
\end{abstract}

\section{Introduction}

It is a well known fact that during long-term steroid therapy an undiagnosed and therefore untreated infection with Myco. tuberculosis may flare up. With this in mind a study was undertaken in 18 leprosy patients on long-term steroid therapy to find out if Lamprene does prevent a flare-up of the infection with Myco. leprae.

\section{Materials and Methods}

The patients were divided in 4 groups:

(1) Patients on daily Lamprene and steroids 12

(2) Patients on bi-weekly Lamprene and steroids 6

(3) Patients on daily Lamprene

(4) Patients on bi-weekly Lamprene

8

33

The total number of patients studied was 49 , all of them LL or BL. Some patients first belonged to one of the 4 groups and later to another. 
Group 4: Lamprene twice weekly $100 \mathrm{mg}$

(a) Thirty patients suffering from reaction (ENL and fever), 8 of whom were found to be suffering from tuberculosis as well.

(b) Three patients with resistance against DDS.

If in spite of bi-weekly Lamprene reactions continued to occur, Lamprene was increased to $100 \mathrm{mg}$ daily.

\section{Group 3: Lamprene daily $100 \mathrm{mg}$}

(a) Seven patients suffering from reaction, 5 of them with concomitant tuberculosis.

(b) One patient with resistance against DDS.

As Lamprene is an expensive drug and not always freely obtainable in India, it was sometimes impossible to increase the dosage to $100 \mathrm{mg}$ daily to suppress reactions. In that case steroids (prednisone) were given according to a standard routine, starting with $40 \mathrm{mg}$ daily and tapering off in 4 weeks to $10 \mathrm{mg}$ daily.

\section{Group 2: Lamprene twice weekly $100 \mathrm{mg}$ and prednisone (40 to $10 \mathrm{mg}$ ) daily}

(a) Four patients with chronic neuritis.

(b) Two patients with chronic reaction, one of them suffering from tuberculosis as well.

If in spite of bi-weekly Lamprene reactions continued to occur, Lamprene was increased to $100 \mathrm{mg}$ daily.

\section{Group 1: Lamprene $100 \mathrm{mg}$ daily and prednisone (40 to) $10 \mathrm{mg}$ daily}

(a) Seven patients with chronic reaction.

(b) Five patients with chronic neuritis.

Seven patients in this group with extremely severe reaction and/or neuritis suffered from tuberculosis as well.

As far as possible bacteriological smears were examined every 3 months and only patients with at least 6 months treatment of the same type and with at least 3 smears were included in the various groups.

\section{Results}

In all patients there was a satisfactory reduction of the MI to less than $1 \%$ and in most cases to zero.

The average decline of the BI was as follows:

Group 1: Daily Lamprene and prednisone: 47\% per annum, varying from 12 to $84 \%$.

Group 2: Bi-weekly Lamprene and prednisone: $54 \%$ per annum, in 1 patient the BI increased by $14 \%$, in the others there was a decline of up to $100 \%$.

Group 3: Daily Lamprene: 25\% per annum, in 2 patients the BI increased by 8 and $10 \%$ respectively, in the others there was a decline of up to $100 \%$.

Group 4: Bi-weekly Lamprene: 37\% per annum, in one patient the BI increased by $5 \%$, in the others there was a decline of up to $100 \%$. 
TABLE 1

Group 1: Daily Lamprene and prednisone

\begin{tabular}{lrcrc}
\hline & $\begin{array}{c}\text { Period } \\
\text { in months }\end{array}$ & $\begin{array}{c}\text { First } \\
\text { smear } \\
\text { BI-Ml }\end{array}$ & $\begin{array}{c}\text { Last } \\
\text { smear } \\
\text { BI-MI }\end{array}$ & $\begin{array}{c}\text { Decline BI } \\
\text { per annum }\end{array}$ \\
\hline 1. M., B L, 1958, chronic neuritis & 18 & $3.3-0.0$ & $1.6-0.0$ & $34 \%$ \\
2. G., BL, 1959, chronic neuritis & 8 & $3.0-0.0$ & $1.5-0.0$ & $75 \%$ \\
3. D., LL, 1963, chronic reaction & 10 & $4.5-0.0$ & $3.5-0.0$ & $27 \%$ \\
4. Y., LL, 1963, chronic reaction, tb & 6 & $4.5-0.3$ & $2.6-0.0$ & $84 \%$ \\
5. K., BL, 1962, chronic neuritis, tb & 8 & $3.1-0.0$ & $2.1-0.0$ & $48 \%$ \\
6. D., LL, 1954, chronic reaction, tb & 19 & $3.5-0.0$ & $1.1-0.0$ & $43 \%$ \\
7. T., LL, 1941, chronic reaction, tb & 13 & $3.1-0.0$ & $1.8-0.0$ & $39 \%$ \\
8. N., BL, 1954, chronic neuritis, tb & 6 & $5.0-0.3$ & $4.3-0.1$ & $28 \%$ \\
9. S., BL, 1954, chronic neuritis & 16 & $2.8-0.0$ & $0.3-0.0$ & $67 \%$ \\
10. L., LL, 1937, chronic reaction & 9 & $2.6-0.0$ & $1.5-0.0$ & $56 \%$ \\
11. S., LL, 1956, chronic reaction, tb & 21 & $3.5-0.0$ & $0.5-0.0$ & $49 \%$ \\
12. P., LL, 1928, chronic reaction, tb & 23 & $4.6-0.3$ & $3.5-0.0$ & $12 \%$ \\
& & & & 562 \\
& & & & \\
\end{tabular}

TABLE 2

Group 2: Bi-weekly Lamprene and prednisone

\begin{tabular}{lccrr}
\hline & $\begin{array}{c}\text { Period } \\
\text { in months }\end{array}$ & $\begin{array}{c}\text { First } \\
\text { smear } \\
\text { BI-MI }\end{array}$ & $\begin{array}{c}\text { Last } \\
\text { smear } \\
\text { BI-MI }\end{array}$ & $\begin{array}{r}\text { Decline BI } \\
\text { per annum }\end{array}$ \\
\hline 1. D., LL, 1961, chronic reaction, tb & 6 & $3.5-0.0$ & $3.0-0.1$ & $29 \%$ \\
2. A., BL, 1954, chronic neuritis & 6 & $3.6-0.0$ & $1.8-0.0$ & $100 \%$ \\
3. I., BL, 1953, chronic neuritis & 8 & $2.1-0.0$ & $2.3-0.2$ & $-14 \%$ \\
4. L., LL, 1937, chronic reaction & 7 & $5.5-0.6$ & $3.8-0.0$ & $53 \%$ \\
5. G., BL, 1957, chronic neuritis & 6 & $3.8-1.2$ & $2.0-0.0$ & $95 \%$ \\
6. S., BL, 1954, chronic neuritis & 9 & $4.1-0.3$ & $2.3-0.0$ & $\frac{59 \%}{322}$ \\
& & & & \\
\end{tabular}

TABLE 3

Group 3: Daily Lamprene

\begin{tabular}{lccrr}
\hline & $\begin{array}{c}\text { Period } \\
\text { in months }\end{array}$ & $\begin{array}{c}\text { First } \\
\text { smear } \\
\text { BI-MI }\end{array}$ & $\begin{array}{c}\text { Last } \\
\text { smear } \\
\text { BI-MI }\end{array}$ & $\begin{array}{r}\text { Decline BI } \\
\text { per annum }\end{array}$ \\
\hline 1. M., LL, 1947, chronic reaction, tb & 7 & $4.8-1.0$ & $4.3-0.0$ & $18 \%$ \\
2. J., LL, 1945, DDS resistant & 6 & $5.0-2.3$ & $4.6-0.3$ & $16 \%$ \\
3. B., LL, 1959, chronic reaction & 8 & $3.1-0.2$ & $2.6-0.0$ & $24 \%$ \\
4. R., LL, 1954, chronic reaction & 7 & $4.3-0.5$ & $4.5-0.2$ & $-8 \%$ \\
5. V., LL, 1961, chronic reaction, tb & 7 & $4.5-0.2$ & $3.6-0.3$ & $34 \%$ \\
6. D. LL., 1961, chronic reaction, tb & 9 & $4.1-0.1$ & $0.3-0.0$ & $124 \%$ \\
7. N., B B, 1954, chronic reaction, tb & 9 & $5.2-3.0$ & $5.1-0.3$ & $3 \%$ \\
8. T., LL, 1941, chronic reaction, tb & 10 & $3.5-0.7$ & $3.8-0.0$ & $-10 \%$ \\
& & & & 201
\end{tabular}

Average: $25 \%$ 
TABLE 4

Group 4: Bi-weekly Lamprene

\begin{tabular}{|c|c|c|c|c|}
\hline & $\begin{array}{l}\text { Period } \\
\text { in months }\end{array}$ & $\begin{array}{c}\text { First } \\
\text { smear } \\
\text { BI-MI } \\
\end{array}$ & $\begin{array}{c}\text { Last } \\
\text { smear } \\
\text { BI-MI } \\
\end{array}$ & $\begin{array}{l}\text { Decline BI } \\
\text { per annum }\end{array}$ \\
\hline 1. R., LL, 1959, chronic reaction & 11 & $2.5-0.3$ & $0.3-0.0$ & $96 \%$ \\
\hline 2. K., LL, 1944, chronic reaction & 12 & $4.1-0.6$ & $4.1-0.2$ & $0 \%$ \\
\hline 3. M., $\mathrm{BL}, 1947$, chronic reaction & 9 & $1.5-0.0$ & $0.0-0.0$ & $133 \%$ \\
\hline 4. R., LL, 1942, chronic reaction, tb & 19 & $4.8-0.0$ & $2.0-0.0$ & $37 \%$ \\
\hline 5. B., LL, 1934, chronic reaction & 7 & $4.1-0.6$ & $3.5-0.3$ & $25 \%$ \\
\hline 6. A., LL, 1937, chronic reaction, tb & 22 & $3.0-0.0$ & $0.0-0.0$ & $55 \%$ \\
\hline 7. S., LL, 1050, chreoic reaction & 28 & $4.5-0.2$ & $0.8-0.0$ & $35 \%$ \\
\hline 8. S., LL, 1949, chronic reaction & 23 & $4.8-0.1$ & $1.6-0.0$ & $35 \%$ \\
\hline 9. J., LL, 1946, chronic reaction & 9 & $5.0-0.1$ & $2.6-0.0$ & $64 \%$ \\
\hline 10. M., LL, 1945 , chronic reaction & 36 & $4.5-8.0$ & $0.6-0.0$ & $29 \%$ \\
\hline 11. B., LL, 1935, chronic reaction & 25 & $5.0-1.0$ & $4.8-0.5$ & $2 \%$ \\
\hline 12. C., LL, 1935, chronic reaction, tb & 7 & $3.8-0.0$ & $2.3-0.0$ & $68 \%$ \\
\hline 13. R., BL, 1951, chronic reaction & 17 & $5.0-0.0$ & $3.8-0.2$ & $17 \%$ \\
\hline 14. Y., LL, 1937, DDS resistant & 22 & $4.6-0.0$ & $3.8-0.1$ & $9 \%$ \\
\hline 15. S., LL, 1930, chronic reaction & 18 & $4.6-0.0$ & $2.1-0.0$ & $36 \%$ \\
\hline 16. P., LL, 1952, chronic reaction & 18 & $3.5-0.0$ & $3.0-0.0$ & $10 \%$ \\
\hline 17. H., LL, 1943, chronic reaction & 27 & $5.3-4.0$ & $1.0-0.0$ & $36 \%$ \\
\hline 18. S., LL, 1947, chronic reaction & 11 & $4.3-0.6$ & $2.1-0.0$ & $56 \%$ \\
\hline 19. V., LL, 1935, DDS resistant & 6 & $4.6-1.5$ & $4.3-0.0$ & $13 \%$ \\
\hline 20. S., BL, 1940, chronic reaction & 7 & $5.0-0.3$ & $4.1-0.1$ & $31 \%$ \\
\hline 21. Y., LL, 1955, chronic reaction & 6 & $5.0-0.5$ & $2.8-0.0$ & $88 \%$ \\
\hline 22. S., LL, 1933, chronic reaction & 7 & $4.0-0.0$ & $3.6-0.0$ & $17 \%$ \\
\hline 23. S., LL, 1943, chronic reaction & 15 & $5.5-0.6$ & $3.3-0.0$ & $32 \%$ \\
\hline 24. M., LL, 1918, DDS resistant & 9 & $4.0-1.1$ & $3.6-0.0$ & $13 \%$ \\
\hline 25. S., LL, 1950, chronic reaction & 15 & $5.0-0.0$ & $2.6-0.0$ & $38 \%$ \\
\hline 26. S., LL, 1954 , chronic reaction, tb & 9 & $5.1-4.0$ & $5.3-1.6$ & $-5 \%$ \\
\hline 27. Y., LL, 1950, chronic reaction, tb & 7 & $3.1-0.0$ & $2.0-0.0$ & $61 \%$ \\
\hline 28. A., BL, 1954, chronic reaction & 15 & $5.0-0.0$ & $2.3-0.0$ & $43 \%$ \\
\hline 29. G., LL, 1939, chronic reaction & 10 & $5.1-0.3$ & $3.5-0.2$ & $38 \%$ \\
\hline 30. P., LL, 1930, chronic reaction & 16 & $5.1-1.5$ & $3.3-0.0$ & $26 \%$ \\
\hline 31. S., LL, 1947, chronic reaction, tb & 28 & $2.1-0.0$ & $0.0-0.0$ & $43 \%$ \\
\hline 32. Y., LL, 1963, chronic reaction, tb & 10 & $5.1-6.0$ & $3.6-0.0$ & $35 \%$ \\
\hline 33. D., LL, 1961, chronic reaction, tb & 8 & $5.4-6.0$ & $5.0-0.2$ & $11 \%$ \\
\hline & & & & 1227 \\
\hline & & & \multicolumn{2}{|c|}{ Average: $37 \%$} \\
\hline
\end{tabular}

\section{Summary}

Group 1: Daily Lamprene and prednisone Group 2: Bi-weekly Lamprene and prednisone Group 3: Daily Lamprene

Group 4: Bi-weekly Lamprene
Average decline $B I$

$47 \%$ (tb patients $43 \%$ ).

$54 \%$ (tb patients $29 \%$ ). $25 \%$ (tb patients $34 \%$ ). $37 \%$ (tb patients $38 \%$ ).

\section{Discussion}

In both groups, with and without prednisone, a few patients showed a slight increase in BI. It is interesting to note that group 1 (daily Lamprene and 
prednisone) seemed to do better than group 3 (daily Lamprene only) and that group 2 (bi-weekly Lamprene and prednisone) seemed to do better than group 4 (bi-weekly Lamprene only).

As the average decline of the $\mathrm{BI}$ in the patients with tuberculosis was about the same as the average decline of BI in the whole group, it does not seem likely that treatment of tuberculosis with streptomycin, $\mathrm{INH}$ and thiosemicarbazone had much influence on the reduction of the $\mathrm{BI}$ in these patients. 